\title{
Recent Advances in Understanding Immunity Against Brucellosis: Application for Vaccine Development
}

\author{
Sérgio Costa Oliveira ${ }^{*}, 1$, Gilson Costa Macedo ${ }^{1}$, Leonardo Augusto de Almeida ${ }^{1}$, \\ Fernanda Souza de Oliveira ${ }^{1}$, Angel Oñate ${ }^{2}$, Juliana Cassataro ${ }^{3}$ and \\ Guillermo Hernán Giambartolomei ${ }^{3}$
}

\author{
${ }^{I}$ Department of Biochemistry and Immunology, Institute of Biological Sciences, Federal University of Minas Gerais, \\ Belo Horizonte-Minas Gerais, Brazil \\ ${ }^{2}$ Department of Microbiology, Faculty of Biological Sciences, Molecular Immunology Laboratory, Universidad de \\ Concepción, Concepción, Chile \\ ${ }^{3}$ Instituto de Estudios de la Inmunidad Humoral (CONICET), Facultad de Farmacia y Bioquímica, Universidad de \\ Buenos Aires (UBA), Buenos Aires, Argentina
}

\begin{abstract}
Brucellosis is an important zoonotic disease of nearly worldwide distribution. This pathogen causes abortion in cattle and undulant fever, arthritis, endocarditis and meningitis in human. The immune response against B. abortus involves innate and adaptive immunity involving antigen-presenting cells, NK cells and CD $4^{+}$and $\mathrm{CD} 8^{+} \mathrm{T}$ cells. IFN- $\gamma$ is a crucial immune component that results from Brucella recognition by host immune receptors such as Toll-like receptors (TLRs) that lead to IL-12 production. Although great efforts to elucidate immunity against Brucella have been employed, the subset of cells and factors involved in host immune response remains not completely understood. Our group and others have been working in an attempt to understand the mechanisms involved in innate responses to Brucella. Understanding the requirements for immune protection can help the design of alternative vaccines that would avoid the drawbacks of currently available vaccines to Brucella. This review discusses recent studies in host immunity to Brucella and new approaches for vaccine development.
\end{abstract}

Keywords: Brucella abortus, vaccines, innate immunity, cytokines, DNA vaccines, recombinant vaccines, deletion mutants.

\section{INTRODUCTION}

Brucella spp. are Gram-negative coccobacilli, aerobic, urease positive, non-motile bacteria which cause brucellosis in humans and in a variety of animal species [1]. Among Brucella species are B. melitensis, B. abortus, B. suis, B. canis, $B$. neotomae, and $B$. ovis. The first four species are pathogenic to humans in decreasing order of severity making brucellosis a zoonotic disease with more than 500,000 new cases reported annually. Brucella spp. can persist in unpasteurized dairy products such as raw milk, soft cheese, butter and ice cream. Additionally, consumption of undercooked animal organs such as spleen and liver has been implicated in human infection [2].

Health and sanitary regulations predicated on fear of spreading virulent cattle diseases, such as brucellosis, have limited the marketing opportunities of cattle products between countries. Despite great regulatory efforts worldwide, pathogenic Brucella spp. can persist in domestic livestock or free-ranging wildlife. Although vaccination is probably the most economic control measure, administration

*Address correspondence to this author at the Laboratory of Immunology of Infectious Diseases, Department of Biochemistry and Immunology, Institute of Biological Sciences, Federal University of Minas Gerais, Belo HorizonteMinas Gerais, 31270-901, Brazil; Tel: +55 31 34092666;

E-mail: scozeus@icb.ufmg.br of currently available vaccines alone is not sufficient for elimination of brucellosis in any host species [3]. Thus, the development of effective vaccines that completely prevent the infection and protect the different hosts of Brucella, is required for elimination of this illness.

As the complete genomic sequences of some Brucella species are available, the search for virulence factors is a key approach to understand the mechanisms used by this bacterium to escape the host immune system. Bioinformatic analysis showed that Brucella lacks classical virulencerelated sequences and genes, such as toxins, type I, II or III secretion systems, pilus biogenesis genes, and others. Our research group has investigated the role of some structural and metabolic components in bacterial pathogenesis using the disrupting gene approach to obtain new attenuated vaccine strains. Additionally, we have dissected some of the pathways involved in Brucella recognition by innate immune receptors using knockout mice.

\section{IMMUNE RESPONSE AGAINST BRUCELLA}

Brucella spp. are facultative intracellular pathogens which resist killing by neutrophilis, replicate inside macrophages and in "non-professional" phagocytes and maintain a long lasting interaction with the host cells [4]. As intracellular organisms, protection against Brucella infection requires cell-mediated immunity, which includes $\mathrm{CD} 4^{+}$and $\mathrm{CD} 8^{+} \mathrm{T}$ lymphocytes, Th1-type cytokines such as IFN- $\gamma$ and 
TNF- $\alpha$, and activated macrophages and dendritic cells (DC) [5]. Therefore, host control of infection requires a set of cells and factors which together promote a complex response against Brucella.

$\mathrm{CD}^{+} \mathrm{T}$ cells have the predominant role for optimal protection against $B$. abortus infection. This protection can be performed by a type 1 cytokine profile production, mainly IFN- $\gamma$, and lysis of Brucella-infected macrophages [6,7]. Lysis of this macrophages releases the bacteria to the extracellular milieu enabling uptake by other activated macrophages in a IFN- $\gamma$-rich microenvironment. These cells presents augmented antibrucellae mechanisms and are able to destruct the pathogen, inhibiting Brucella spread [8]. Moreover, the type 1 cytokines produced by $\mathrm{CD}^{+} \mathrm{T}$ cells induce down-regulation of Th2 cytokines and IL-10 [6, 7].

Since mice can develop the symptoms of brucellosis when infected intraperitonially, they are widely used as experimental model to better understand the infection course, even though mice are not considered natural host of Brucella. The infection process in BALB/c and C57BL/10 mice differs in their abilities to induce immune responses and protection against virulent Brucella [9-11]. BALB/c mice are more sensitive than $\mathrm{C} 57 \mathrm{BL} / 6$ mice to virulent Brucella such as B. melitensis, B. abortus, and B. suis. The infection control correlates with the level of IFN- $\gamma$ produced by $\mathrm{CD}^{+} \mathrm{T}$ cells at specific times following infections in these mouse models [9]. Sathiyaseelan et al. (2006) [12] demonstrated that BALB/c mice produce IFN- $\gamma$ in the first week of infection with B. abortus, however the level of IFN$\gamma$ production decreases and this is consistent with transiently decrease of IL-12R $\beta 2^{+}$cells during the course of brucellosis. Also, the administration of recombinant IL-12 restored the ability of BALB/c splenocytes to produce IFN- $\gamma$ and increases protective immunity. As IFN- $\gamma$ produced by T cells is vital for control of infection, Brucella organisms have to face a second challenge if willing to persist in the host for a long time. Bacteria should be able to inhibit Ag processing and presentation by Brucella-containing macrophages to avoid the immunological surveillance of MHC-II restricted IFN- $\gamma$-producing CD4+ $\mathrm{T}$ lymphocytes. Recently, Barrionuevo et al. (2008) [13] have demonstrated that infection with Brucella species down-modulates expression of MHC-II and Ag presentation on monocytes/macrophages. This phenomenon was induced by L-Omp19, a prototypical Brucella lipoprotein and it was dependent of TLR-2 and it was mediated by IL-6.

Brucella species, such as B. suis or B. melitensis, are able to induce chronic infection and multiply inside human macrophages. Bessoles et al. (2009) [14] demonstrated that intramacrophagic Brucella multiplication is impaired in presence of $\mathrm{CD}^{+}{ }^{+} \mathrm{NNKTcells.} \mathrm{The} \mathrm{impairment} \mathrm{of} \mathrm{Brucella}$ growth by $\mathrm{CD} 4{ }^{+}$iNKT cells requires an interaction with CD1d present on macrophage surface. These authors suggested that $\mathrm{CD} 4{ }^{+} \mathrm{iNKT}$ cells can contribute to host control of infection at several levels, indirectly by influencing the development of adaptive immune response through the production of cytokines and directly on the clearance of bacteria through eliminating infected cells and/or killing intracellular bacteria. Also related with the impairment of Brucella growth are the T cells of the $\gamma \delta$ subset. These cells are capable of inhibiting Brucella growth through a combination of mechanisms such as cytotoxicity, macrophage activation through cytokine and chemokine secretion and antibacterial effects [15]. Besides, inducing macrophage death and reduction of intracellular Brucella through Fas-FasL interactions, the $\mathrm{V} \gamma 9 \mathrm{~V} \delta 2 \mathrm{~T}$ cells, the major subtype of $\gamma \delta \mathrm{T}$ cells, also release soluble factors responsible for a bactericidal activity of these cells limiting the spread of this intracellular pathogen $[15,16]$.

Brucella is also able to survive inside human neutrophils $[17,18]$. Furthermore, B. abortus induced an increase in $\mathrm{CD} 35$ and $\mathrm{CD} 11 \mathrm{~b}$ expression and a decline in CD62L accompanied by IL-8 secretion, a response compatible with neutrophil activation which can lead to tissue damage and pathology associated with human brucellosis. This neutrophil activation also is induced by stimulation with LOmp19, which is a Brucella lipoprotein. Thus, Brucella lipoproteins possess pro-inflammatory properties that could contribute to tissue injury and inflammation by direct activation of neutrophils [19].

\subsection{The Role of Toll-Like Receptors in Brucella abortus Infection}

The first line of defensive mechanisms begins with the recognition of pathogen-associated molecular patterns (PAMPs) by pattern recognition receptors (PRRs) [20]. There are several functionally distinct classes of PRRs. The best characterized are the Toll-like receptors (TLRs), which are transmembrane receptors that sense lipids, lipoproteins, proteins and nucleic acids [21]. Recognition of PAMPs by TLRs stimulates the recruitment of a set of intracellular TIRdomain-containing adaptors, including MyD88, TIRAP, TRIF and TRAM via TIR-TIR interactions to initiate signaling. MyD88 initiates a cascade that leads to the activation of MAP kinases (MAPKs: ERK, JNK, p38) and the transcription factor NF-kB to control the expression of inflammatory cytokines genes. TIRAP mediates the activation of a MyD88-dependent pathway downstream of TLR2 and TLR4. Alternatively, TLR3 and TLR4 may recruit TRIF and activate another pathway (TRIF-dependent pathway) that leads to the activation of the transcription factor IRF3 and involves the production of type I IFN, particularly IFN- $\beta$. TRAM selectively participates in the activation of the TRIF-dependent pathway downstream of TLR4, but not TLR3. Therefore, each TLR recruits a specific set of TIR domain-containing adapters, which in turn triggers different transcription factors, controlling innate immune responses and further leading to the development of antigen-specific acquired immunity [22].

Our group has evaluated the role of a number of TLRs and its adaptor molecules as well as cytokine receptors involved in innate immune responses that might be critical in controlling B. abortus. Regarding the ability of host TLRs to recognize Brucella, there are some contradictory data reported in the literature. We have reported a role for TLR2 and TLR4 on Brucella signaling but we only observed the involvement of TLR4 in resistance [23]. Barquero-Calvo et al. (2007) [24] observed that TNF- $\alpha$ secretion in macrophages culture infected with $B$. abortus seems to depend somewhat on TLR2 and TLR4, but the signaling by these receptors does not affect the intracellular replication of this pathogen. However, when both TLRs were absent, it 
was observed a partially dependence of these molecules, suggesting that signaling by at least one of these receptors is required to prolong host survival. Regarding TLR2, Giambartolomei et al. [25] have demonstrated that $B$. abortus lipoproteins Omp16 and Omp19 induced the production of pro- and anti-inflammatory cytokines in THP1 cell line and this cell activation is TLR2-dependent. Brucella Omp19 lipoprotein also has been found to induce the expression of IL-12 and other pro-inflammatory cytokines and up-regulation of cell surface markers in monocytes/macrophages and DC. The cytokine released by DC, including IL-12, was also dependent on TLR2 stimulation [26]. Biological responses induced by Brucella lipoproteins were dependent on their lipid moiety since unlipidated Omp19 and Omp16 were unable to stimulate cellular responses. Additionally, we have reported that in murine DC HKBA-induced IL-12 production was TLR9dependent [27]. It may be also related with the premise that TLR9 is the relevant TLR for controlling Brucella infection through specialized dendritic cells acting in concert with other cells for generating IFN- $\gamma$ [28]. Coppin et al. [28] suggested that TLR4 cooperates with TLR9 in Brucella detection. Despite the discrepancy on the data demonstrating TLR4-dependent innate responses during Brucella clearance in vivo, TLR9 has been shown to be the TLR that plays a more prominent role during infection [27].

The molecule MyD88, which is the adaptor molecule for several TLRs and some interleukin receptors, is clearly required for the control of Brucella replication in mice [2729], suggesting that signaling through receptors that use MyD88 is critical to control brucellosis. Our group has investigated the mechanisms involved in MyD88 KO susceptibility and has demonstrated that these animals present impairment on maturation of DC and absence of IL12 and TNF- $\alpha$ production by macrophage and DC in response to heat killed B. abortus (HKBa). IL-12 was shown to be critical to this susceptibility as soon as the treatment with a recombinant virus expressing IL-12 enhances MyD88 $\mathrm{KO}$ resistance to Brucella [27]. MyD88 is also used by other inflammatory signaling pathways such as IL-1 and IL-18; however, signaling through IL-1R or IL-18R seems to be not necessary for host defense against Brucella infection (unpublished results; [29]).

\section{Recent Approaches in Brucella Vaccine Development}

The development of an effective vaccine against brucellosis has been a challenge to scientists around the world. According to Adams (1990) [30], an ideal vaccine against Brucella should have the following requirements: i) prevents the bacterium infection in both gender; ii) not provoke disease in vaccinated animals; iii) prevents abortion; iv) promotes long period of protection with only one dose; v) not interfere with serological diagnosis; vi) biologically stable and should not present risk of virulence reversion; vii) not be pathogenic to humans and should not contaminate products derivate from the vaccinated animals; and, besides, viii) it should be produced in large scale.

At moment, three Brucella strains have been used in brucellosis prevention: S19, Rev1 e RB51. However, these strains are still far from ideal. Although the smooth strains S19 from B. abortus and Rev1 from B. melitensis are able to induce effective levels of protection in cattle and in goat and sheep; respectively, they have some problems. These vaccine strains can cause abortion in pregnant animals and they can be secreted in milk of vaccinated animals. Besides, both of them are pathogenic to humans and interferes with the diagnosis because they possess the LPS contained the intact $\mathrm{O}$-chain. The antibody production against $\mathrm{O}$-chain troubles the differentiation between vaccinated from infected animals [31]. On the other hand, the mutant strain RB51 derivated from $B$. abortus, does not possess intact O-chain which avoid interference in serological diagnosis. Besides, RB51 is stable and is less virulent then the smooth strains [32]. However, this strain is resistant to rimfampicin that is the first antibiotic of choice to human brucellosis treatment [33]. Moreover, the immunization effectiveness of RB51 is controversial in different hosts [34].

To solve the problems presented by the currently available vaccines, several efforts have been performed to improve these immunogens. In an attempt to increase the protection given by the rough vaccine strains, Grilló and colleagues (2006) [35] demonstrated that the coadministration of different Brucella mutants can confers protection against murine brucellosis. In that study, the investigators produced mutants to O-chain, specifically to wbkA gene, and to two components regulatory system $b v r S / b v r R$. The vaccination combining the two mutants conferred better levels of protection when compared to the S19 protection. Also, the combination of rough mutants was not able to induce the production of antibodies against the Ochain as the S19 vaccine strain S19.

Arenas-Gamboa et al. (2009) [36], using the vaccine strain S19, developed a mutant to $v j b R$ gene, which encodes a transcriptional regulator associated to $\operatorname{vir} B$ expression, and it is associated to bacterium virulence and bacterium surveillance inside macrophages. This potential vaccine was delivered in microcapsules as a different delivery system. They reported higher level of protection induced by this vaccination strategy when compared to S19 strain. Besides, inflammation and persistence was also decreased.

Izadjoo and coworkers (2004) [37] evaluated a orally administered live attenuated purine-auxotrophic Brucella melitens mutant strain, WR201. The ability of this mutant strain to elicit cellular and humoral immune responses and to protect mice against intranasal challenge with $B$. melitensis $16 \mathrm{M}$ was evaluated. In this report, the strain WR201 was able to induce cellular, humoral and mucosal immune responses. Moreover, oral immunization induced protection against systemic bacterial spread and enhanced clearance of bacteria from the lungs after intranasal challenge. These results suggest that purine auxotrophy is an attractive attenuating strategy for further vaccine development. However, in another study, these researchers showed that WR201 mutant retains its infectivity for reproductive tissues [38]. This tropism may lead to signs and symptoms of disease in man.

In attempt to construct a vaccine against Brucella ovis infection which was not capable to interfere in differentiation between vaccinated from infected animals, Grilló et al. (2009) [39] tested the deletion of bp26 gene (CGV26) or both bp26 and omp31 genes (CGV2631) in B. melitensis Rev 1. Bp26 and Omp31 are two proteins that have potential 
differential diagnostic interest [40-42]. After subcutaneous administration in rams, the mutants conferred significant protection; however, the level of protection induced by CGV26 was higher than that conferred by CGV2331 and similar to that engendered by Rev.1. These results demonstrated that the CGV26 mutant, associated with an adequate diagnostic strategy, could be a useful alternative to Rev.1 reducing the problem of serological interferences.

Since RB51 does not confer resistance to B. melitensis or $B$. ovis, other rough strains have been evaluated as potential vaccine. Recently, Adone and colleagues (2008) [43] described that vaccination with the rough mutant strain B115 from $B$. melitensis engendered significant levels of protection against these species of Brucella in BALB/c mice. Even though this mutant can produce cytoplasmatic levels of O-chain, antibodies against LPS were not detected.

The use of Brucella specific antigens as potential vaccine candidates has been also exhaustively investigated. The most used strategy is the identification of antigens able to induce a strong cellular immune response [44]. In this regard, some antigens have induced interesting results such as the protein p39 [45], the $\mathrm{Cu}-\mathrm{Zn}$ superoxide dismutase [46], the ribosomal protein $\mathrm{L} 7 / \mathrm{L} 12$ [47-50], the heat shock proteins GroEL and GroES [47], the lumazine synthase [51], Omp-31 [52] and the glyceraldehyde-3-phosphate-dehydrogenase [53].

Recently, it was demonstrated that immunization with non-lipidated (U) forms of recombinant membrane proteins Omp-16 and Omp-19 (U-Omp16 or U-Omp19) induced a $\mathrm{T}_{\mathrm{H}} 1$ response, systemic protection in aluminum hydroxide formulation, and oral protection with cholera toxin adjuvant against $B$. abortus infection. Both immunization routes exhibited a similar degree of protection to attenuated Brucella vaccines (S19 and RB51, respectively). These results suggest the possible use of these proteins for a subunit vaccine against human and animal brucellosis [54].

The recombinant proteins SurA (a periplasmic peptidyl prolyl cis-trans isomerase) and DnaK (a chaperone from HSP70 family) were also evaluated. These proteins were able to induce a robust humoral response, IFN- $\gamma$ and a cytotoxic response. However, the protection level induced by these proteins is lower than the protection conferred by the live vaccine $\mathrm{S} 19$ [55].

Mallic and colleagues (2007) [56], in an attempt to improve the immunogenicity of Brucella antigens, demonstrated that the recombinant liposomized protein L7/L12 could induced strong cell immune response with increase of $\mathrm{T}$ cell proliferation, production of $\mathrm{T}_{\mathrm{H}} 1$ cytokines and it could induced a strong humoral immune response. This strategy was also effective in improving the bacteria clearance after challenge with $B$. abortus 544, inducing protection levels comparable to S19 vaccine.

Live genetically modified microorganisms have been used as vector to stimulate host immune system recently. Recently, Harms and colleagues (2009) [57] developed a modified E. coli expressing invasion of Yersinia and listerialysin O (LLO) of Listeria capable to infect a range of cell types and to release Brucella antigens inside the host cell. They presented a vaccine vector that mimics the Brucella intracellular infection which induces the differentiation to a $T_{\mathrm{H}} 1$ immune response and stimulates specific cytolytic T lymphocytes (CTLs).

As the gastrointestinal tract seems to be one of the main entrance to Brucella infection [58], Zhao and coworkers (2009) [59], used Salmonela enterica serovar Typhimurium, as a vaccine vector. When administrated orally, the vector is capable to express the ribosomal protein $\mathrm{L} 7 / \mathrm{L} 12$ and the lumazine synthase enzyme (BLS). This treatment was able to stimulate the mucosal immunity and $\mathrm{T}_{\mathrm{H}} 1$ mediated immunity. However, the levels of protection after B. abortus 544 challenge were lower than those encountered in vaccination with attenuated strain B. abortus $104 \mathrm{M}$.

Cabrera and colleagues (2009) [60] constructed an infectious but replication-deficient Semliki Forest virus (SFV) particles carrying recombinant RNA encoding the Brucella abortus translation initiation factor 3 (IF3). $\mathrm{BALB} / \mathrm{c}$ mice immunized with SFV-IF3 exhibited a significant level of resistance against challenge with the virulent $B$. abortus S2308, similarly to immunization with the live RB51 vaccine strain. Furthermore, SFV-IF3 immunization induced a $\mathrm{T}_{\mathrm{H}} 1$ biased immune response with increased levels of IFN- $\gamma$ and low levels of IL-4. These findings demonstrated the potential use this immunization approach to induce protection against Brucella infection.

DNA vaccines are considered an important strategy of vaccination that has been extensively investigated due the capacity to induce humoral and cell immune response [61]. Different Brucella genes have been evaluated through DNA vaccination $[45,51,62-65]$. However, until now, no monovalent DNA vaccine have demonstrated superior efficacy when compared to commercial vaccines [66]. In an attempt to produce an effective DNA vaccine against brucellosis, Luo et al. (2006) [67], developed a divalent DNA vaccine which codes for L7/L12 and Omp-16 proteins from $B$. abortus. As expected, this vaccine was able to induce a robust cell immune response with high $\mathrm{T}$ cell proliferation and IFN- $\gamma$ production compared to Omp-16 or L7/L12 monovalent DNA vaccine, suggesting that these genes, together, can be a target for DNA vaccines. Also, the protection levels induced by Omp-16/L7L12 DNA vaccine were higher than those induced by monovalent preparations. However, the immunization with divalent Omp-16/ L7L12 can still provide less protection than the attenuated rough strain B. abortus RB51.

Cassataro et al. (2007) [68] demonstrated that a chimera with the scaffold protein BLS decorated with ten copies of a $\mathrm{B}$ and Th1 epitope derived from the Omp31 protein induced similar protection than Rev.1 vaccination against $B$. ovis infection. After this, they investigated the immunogenicity and protective capacity of the chimera as a DNA vaccine. Their results demonstrated that the addition of an immunodominant epitope to the N-termini of BLS significantly improved the degree of protection achieved by each immunogen individually. In contrast, immunization with both antigens (pcDNABLS and pCIOmp31) showed no additive or synergic effect on protection compared with the use of single antigen (Ags), independently of the immunization system used (DNA or recombinant protein immunization). Strikingly, the chimera as a DNA vaccine induced significantly higher protection against $B$. ovis than the Rev.1 vaccine, which contains all Ags of Brucella spp. 
The chimera as a DNA vaccine also induced statistically similar levels of protection than the control vaccine Rev.1 against $B$. melitensis. Moreover, this vaccine also elicited long term protection against smooth and rough Brucella tested at 5 months after immunization [69].

Additionally, Yu and coworkers (2007) [66], constructed a combined DNA vaccine containing genes that codes for BCSP31, superoxide dismutase and L7/L12 antigens. The immunization with this group of genes stimulated a strong humoral immune response with higher induction of specific IgG, considerable IFN- $\gamma$ and TNF- $\alpha$ production and the accumulation of $\mathrm{CD} 8^{+} \mathrm{T}$ lymphocytes. This combined vaccine was able to induce superior levels of protection when compared to live vaccine strains (S19 and RB51), suggesting the use of it in immunization of large animals. In another interesting study, $\mathrm{Hu}$ and coworkers (2009) [70] demonstrated that a combined DNA vaccine protects cattle against two infectious diseases, Brucellosis and Tuberculosis. The researchers constructed a DNA vaccine containing six genes encoding immunodominant antigens from Mycobacterium bovis (Ag85B, MPT64 and MPT83) and Brucella abortus (BCSP31, SOD and L7/L12). Vaccination with this preparation induced higher serum concentrations of both IFN- $\gamma$ and IgG antibodies in addition to the improved $\mathrm{CD}^{+} \mathrm{T}$ cell-responses. Moreover, significantly enhancement of protection was observed comparing the BCG vaccine against $M$. bovis and protection levels of S19 vaccine strain against $B$. abortus.

\section{CONCLUDING REMARKS}

Brucellosis, in particular infections with Brucella abortus, Brucella melitensis or Brucella suis, remains a significant human health threat in many areas of the world. Brucella is recognized by host cells via TLR2, TLR4, and TLR9. However, TLR9 seems to be the most important innate immune receptor in controlling Brucella infection in vivo. Regarding cellular immunity, $\mathrm{CD}^{+}$and $\mathrm{CD}^{+} \mathrm{T}$ cells are important subsets involved in protection. However, our group has reported the more prominent role of $\mathrm{CD} 8^{+}$over $\mathrm{CD}^{+} \mathrm{T}$ lymphocytes and this might be due to the intracellular localization of Brucella. More recently, NKT and $\gamma \delta \mathrm{T}$ cells were also identified as important components of host immune response. The prevention of human brucellosis predominantly depends on the eradication of the disease in their primary hosts. Currently used programs to eradicate brucellosis in cattle, sheep and goats rely on live attenuated vaccines. The virulence of those vaccine strains is nevertheless often high enough to cause abortion if administered to pregnant animals, and may be infectious for persons handling and administering the vaccine. Therefore, the development of effective vaccines that successfully protect the different hosts of Brucella without interfering with serodiagnostic remains a challenge. Additionally, there is a feeling among Brucella scientists that a genetically engineered live-attenuated vaccine might be the best option to replace current available commercial vaccines. However, we have to be aware of the difficulties regarding approval of such vaccine in regulatory agencies worldwide.

\section{ACKNOWLEDGEMENTS}

This work was supported by grants from FAPEMIG, CNPq, CNPq/Prosul (\#490485/2007-3), CNPq/ANPCy (\# 490528/2008-2), and INCT-Vacinas (CNPq/FAPEMIG).

\section{REFERENCES}

[1] Corbel MJ, Morgan WJB. Genus Brucella Meyer and Shaw. In: Holt JG, Ed. Bergey's Manual of Systematic Bacteriology 1984.

[2] Pappas G, Akritidis N, Bosilkovski M, et al. Brucellosis. N Engl J Med 2005; 352(22): 2325-36.

[3] Olsen SC, Stoffregen WS. Essential role of vaccines in brucellosis control and eradication programs for livestock. Expert Rev Vaccines 2005; 4: 915-28.

[4] Dornand J, Gross A, Lafont V, et al. The innate immune response against Brucella in humans. Vet Microbiol 2002; 90: 383-94.

[5] Golding B, Scott DE, Scharf O, et al. Immunity and protection against Brucella abortus. Microbes Infect 2001; 3: 43-8.

[6] Oliveira SC, Harms JS, Rech EL, et al. The role of T cell subsets and cytokines in the regulation of intracellular bacterial infection. Braz J Med Biol Res 1998; 31: 77-84.

[7] Oliveira SC, Splitter GA. CD8+ type 1 CD44hi CD45 RBlo T lymphocytes control intracellular Brucella abortus infection as demonstrated in major histocompatibility complex class I- and class II-deficient mice. Eur J Immunol 1995; 25: 2551-7.

[8] Jiang X, Baldwin CL. Effects of cytokines on intracellular growth of Brucella abortus. Infect Immun 1993; 61: 124-34.

[9] Fernandes DM, Jiang X, Jung JH, et al. Comparison of T cell cytokines in resistant and susceptible mice infected with virulent Brucella abortus strain 2308. FEMS Immunol Med Microbiol 1996; 16: 193-203.

[10] Li HZ. A pathohistological study on experimental brucellosis in inbred mice. Zhonghua Bing Li Xue Za Zhi. 1991; 20: 134-6.

[11] Montaraz JA, Winter AJ. Comparison of living and nonliving vaccines for Brucella abortus in BALB/c mice. Infect Immun 1986; 53: 245-51.

[12] Sathiyaseelan J, Goenka R, Parent M, et al. Treatment of Brucellasusceptible mice with IL-12 increases primary and secondary immunity. Cell Immunol 2006; 243: 1-9.

[13] Barrionuevo P, Cassataro J, Delpino MV, et al. Brucella abortus inhibits major histocompatibility complex class II expression and antigen processing through interleukin-6 secretion via Toll-like receptor 2. Infect Immun 2008; 76: 250-62.

[14] Bessoles S, Dudal S, Besra GS, et al. Human CD4+ invariant NKT cells are involved in antibacterial immunity against Brucella suis through CD1d-dependent but CD4-independent mechanisms. Eur J Immunol 2009; 39: 1025-35.

[15] Dudal S, Turriere C, Bessoles S, et al. Release of LL-37 by activated human Vgamma9Vdelta2 T cells: a microbicidal weapon against Brucella suis. J Immunol 2006; 177: 5533-9.

[16] Oliaro J, Dudal S, Liautard J, et al. Vgamma9Vdelta2 T cells use a combination of mechanisms to limit the spread of the pathogenic bacteria Brucella. J Leukoc Biol 2005; 77: 652-60.

[17] Riley LK, Robertson DC. Ingestion and intracellular survival of Brucella abortus in human and bovine polymorphonuclear leukocytes. Infect Immun 1984; 46: 224-30.

[18] Kreutzer DL, Dreyfus LA, Robertson DC. Interaction of polymorphonuclear leukocytes with smooth and rough strains of Brucella abortus. Infect Immun 1979; 23: 737-42.

[19] Zwerdling A, Delpino MV, Pasquevich KA, et al. Brucella abortus activates human neutrophils. Microbes Infect 2009; 11: 689-97.

[20] Kawai T, Akira S. TLR signaling. Semin Immunol 2007; 19: 2432 .

[21] Medzhitov R. Recognition of microorganisms and activation of the immune response. Nature 2007; 449: 819-26.

[22] Kawai T, Akira S. Signaling to NF-kappaB by Toll-like receptors. Trends Mol Med 2007; 13: 460-9.

[23] Campos MA, Rosinha GM, Almeida IC, et al. Role of Toll-like receptor 4 in induction of cell-mediated immunity and resistance to Brucella abortus infection in mice. Infect Immun 2004; 72: 176-86. 
[24] Barquero-Calvo E, Chaves-Olarte E, Weiss DS, et al. Brucella abortus uses a stealthy strategy to avoid activation of the innate immune system during the onset of infection. PLoS One 2007; 2: 631.

[25] Giambartolomei GH, Zwerdling A, Cassataro J, et al. Lipoproteins, not lipopolysaccharide, are the key mediators of the proinflammatory response elicited by heat-killed Brucella abortus. J Immunol 2004; 173: 4635-42.

[26] Zwerdling A, Delpino MV, Barrionuevo $\mathrm{P}$, et al. Brucella lipoproteins mimic dendritic cell maturation induced by Brucella abortus. Microbes Infect 2008; 10: 1346-54.

[27] Macedo GC, Magnani DM, Carvalho NB, et al. Central role of MyD88-dependent dendritic cell maturation and proinflammatory cytokine production to control Brucella abortus infection. J Immunol 2008; 180: 1080-7.

[28] Copin R, De Baetselier P, Carlier Y, et al. MyD88-dependent activation of B220-CD11b+LY-6C + dendritic cells during Brucella melitensis infection. J Immunol 2007; 178: 5182-91.

[29] Weiss DS, Takeda K, Akira S, et al. MyD88, but not toll-like receptors 4 and 2, is required for efficient clearance of Brucella abortus. Infect Immun 2005; 73: 5137-43.

[30] Adams LG. Development of live Brucella vaccines. In: Adams LG, ed. Advances in brucellosis research. Texas: A \& M University Press 1990; pp. 205-76.

[31] Nicoletti P. Vaccination. In: Nielsen K, Dunca JR, Eds. Animal Brucellosis. Boca Raton: CRC Press 1990; pp. 284-99.

[32] Schurig GG, Roop RM 2nd, Bagchi T, et al. Biological properties of RB51; a stable rough strain of Brucella abortus. Vet Microbiol 1991; 28: 171-88.

[33] Ariza J. Brucellosis: an update. The perspective from the Mediterranean basin. Rev Med Microbiol 1999; 10: 125-35.

[34] Moriyon I, Grillo MJ, Monreal D, et al. Rough vaccines in animal brucellosis: structural and genetic basis and present status. Vet Res 2004; 35: 1-38.

[35] Grillo MJ, Manterola L, de Miguel MJ, et al. Increases of efficacy as vaccine against Brucella abortus infection in mice by simultaneous inoculation with avirulent smooth bvrS/bvrR and rough wbkA mutants. Vaccine 2006; 24: 2910-6.

[36] Arenas-Gamboa AM, Ficht TA, Kahl-McDonagh MM, et al. The Brucella abortus S19 DeltavjbR live vaccine candidate is safer than S19 and confers protection against wild-type challenge in BALB/c mice when delivered in a sustained-release vehicle. Infect Immun 2009; 77: 877-84.

[37] Izadjoo MJ, Bhattacharjee AK, Paranavitana CM, et al. Oral vaccination with Brucella melitensis WR201 protects mice against intranasal challenge with virulent Brucella melitensis 16M. Infect Immun 2004; 72: 4031-9.

[38] Izadjoo MJ, Mense MG, Bhattacharjee AK, et al. A study on the use of male animal models for developing a live vaccine for brucellosis. Transbound Emerg Dis 2008; 55(3-4): 145-51.

[39] Grillo MJ, Marin CM, Barberan M, et al. Efficacy of bp26 and bp26/omp31 B. melitensis Rev.1 deletion mutants against Brucella ovis in rams. Vaccine 2009; 27:187-91.

[40] Cassataro J, Pasquevich K, Bruno L, et al. Antibody reactivity to Omp31 from Brucella melitensis in human and animal infections by smooth and rough Brucellae. Clin Diagn Lab Immunol 2004; 11(1): 111-4.

[41] Zygmunt MS, Baucheron S, Vizcaino N, et al. Single-step purification and evaluation of recombinant BP26 protein for serological diagnosis of Brucella ovis infection in rams. Vet Microbiol 2002; 87(3): 213-20.

[42] Cloeckaert A, Baucheron S, Vizcaino N, et al. Use of recombinant BP26 protein in serological diagnosis of Brucella melitensis infection in sheep. Clin Diagn Lab Immunol 2001; 8(4): 772-5.

[43] Adone R, Francia M, Ciuchini F. Evaluation of Brucella melitensis $\mathrm{B} 115$ as rough-phenotype vaccine against $B$. melitensis and $B$. ovis infections. Vaccine 2008; 26: 4913-7.

[44] Schurig GG, Sriranganathan N, Corbel MJ. Brucellosis vaccines: past, present and future. Vet Microbiol 2002; 90(1-4): 479-96.

[45] Al-Mariri A, Tibor A, Mertens P, et al. Protection of BALB/c mice against Brucella abortus 544 challenge by vaccination with bacterioferritin or P39 recombinant proteins with $\mathrm{CpG}$ oligodeoxynucleotides as adjuvant. Infect Immun 2001; 69: 481622 .
[46] Tabatabai LB, Pugh GW Jr. Modulation of immune responses in $\mathrm{Balb} / \mathrm{c}$ mice vaccinated with Brucella abortus $\mathrm{Cu}-\mathrm{Zn}$ superoxide dismutase synthetic peptide vaccine. Vaccine 1994; 12: 919-24.

[47] Oliveira SC, Splitter GA. Immunization of mice with recombinant L7/L12 ribosomal protein confers protection against Brucella abortus infection. Vaccine 1996; 14: 959-62.

[48] Oliveira SC, Zhu Y, Splitter G. Sequences of the rplJL operon containing the L10 and L7/L12 genes from Brucella abortus. Gene 1994; 140: 137-8.

[49] Oliveira SC, Zhu Y, Splitter GA. Recombinant L7/L12 ribosomal protein and gamma-irradiated Brucella abortus induce a T-helper 1 subset response from murine CD4+ T cells. Immunology 1994; 83: 659-64.

[50] Oliveira SC, Harms JS, Banai M, et al. Recombinant Brucella abortus proteins that induce proliferation and gamma-interferon secretion by CD4+ $\mathrm{T}$ cells from Brucella-vaccinated mice and delayed-type hypersensitivity in sensitized guinea pigs. Cell Immunol 1996; 172: 262-8.

[51] Velikovsky CA, Cassataro J, Giambartolomei GH, et al. A DNA vaccine encoding lumazine synthase from Brucella abortus induces protective immunity in BALB/c mice. Infect Immun 2002; 70: 2507-11.

[52] Cassataro J, Estein SM, Pasquevich KA, et al. Vaccination with the recombinant Brucella outer membrane protein 31 or a derived 27amino-acid synthetic peptide elicits a CD4+ $\mathrm{T}$ helper 1 response that protects against Brucella melitensis infection. Infect Immun 2005; 73: 8079-88.

[53] Rosinha GM, Myioshi A, Azevedo V, et al. Molecular and immunological characterisation of recombinant Brucella abortus glyceraldehyde-3-phosphate-dehydrogenase, a T- and B-cell reactive protein that induces partial protection when coadministered with an interleukin-12-expressing plasmid in a DNA vaccine formulation. J Med Microbiol 2002; 51: 661-71.

[54] Pasquevich KA, Estein SM, Garcia Samartino C, et al. Immunization with recombinant Brucella species outer membrane protein Omp16 or Omp19 in adjuvant induces specific CD4+ and CD8 + $\mathrm{T}$ cells as well as systemic and oral protection against Brucella abortus infection. Infect Immun 2009; 77: 436-45.

[55] Delpino MV, Estein SM, Fossati CA, et al. Vaccination with Brucella recombinant DnaK and SurA proteins induces protection against Brucella abortus infection in BALB/c mice. Vaccine 2007; 25: 6721-9.

[56] Mallick AI, Singha H, Chaudhuri P, et al. Liposomised recombinant ribosomal $\mathrm{L} 7 / \mathrm{L} 12$ protein protects $\mathrm{BALB} / \mathrm{c}$ mice against Brucella abortus 544 infection. Vaccine 2007; 25: 3692 704.

[57] Harms JS, Durward MA, Magnani DM, et al. Evaluation of recombinant invasive, non-pathogenic Eschericia coli as a vaccine vector against the intracellular pathogen, Brucella. J Immune Based Ther Vaccines 2009; 7: 1.

[58] Gorvel JP, Moreno E, Moriyon I. Is Brucella an enteric pathogen? Nat Rev Microbiol 2009; 7: 250; author reply

[59] Zhao Z, Li M, Luo D, et al. Protection of mice from Brucella infection by immunization with attenuated Salmonella enterica serovar typhimurium expressing A L7/L12 and BLS fusion antigen of Brucella. Vaccine 2009; 27: 5214-9.

[60] Cabrera A, Saez D, Cespedes S, et al. Vaccination with recombinant Semliki Forest virus particles expressing translation initiation factor 3 of Brucella abortus induces protective immunity in BALB/c mice. Immunobiology 2009; 214(6): 467-74.

[61] Leclercq S, Harms JS, Oliveira SC. Enhanced efficacy of DNA vaccines against an intracellular bacterial pathogen by genetic adjuvants. Curr Pharm Biotechnol 2003; 4: 99-107.

[62] Kurar E, Splitter GA. Nucleic acid vaccination of Brucella abortus ribosomal L7/L12 gene elicits immune response. Vaccine 1997; 15: 1851-7.

[63] Onate AA, Cespedes S, Cabrera A, et al. A DNA vaccine encoding $\mathrm{Cu}, \mathrm{Zn}$ superoxide dismutase of Brucella abortus induces protective immunity in BALB/c mice. Infect Immun 2003; 71: 4857-61.

[64] Mayfield JE, Bricker BJ, Godfrey H, et al. The cloning, expression, and nucleotide sequence of a gene coding for an immunogenic Brucella abortus protein. Gene 1988; 63: 1-9.

[65] Cassataro J, Velikovsky CA, de la Barrera S et al. A DNA vaccine coding for the Brucella outer membrane protein 31 confers protection against $B$. melitensis and $B$. ovis infection by eliciting a specific cytotoxic response. Infect Immun 2005; 73: 6537-46. 
[66] Yu DH, Hu XD, Cai H. A combined DNA vaccine encoding BCSP31, SOD, and L7/L12 confers high protection against Brucella abortus 2308 by inducing specific CTL responses. DNA Cell Biol 2007; 26: 435-43.

[67] Luo D, Ni B, Li P, et al. Protective immunity elicited by a divalent DNA vaccine encoding both the L7/L12 and Omp16 genes of Brucella abortus in BALB/c mice. Infect Immun 2006; 74: 273441.

[68] Cassataro J, Pasquevich KA, Estein SM, et al. A recombinant subunit vaccine based on the insertion of 27 amino acids from
Omp31 to the N-terminus of BLS induced a similar degree of protection against $B$. ovis than Rev.1 vaccination. Vaccine 2007; 25: 4437-46.

[69] Cassataro J, Pasquevich KA, Estein SM, et al. A DNA vaccine coding for the chimera BLSOmp31 induced a better degree of protection against $B$. ovis and a similar degree of protection against B. melitensis than Rev.1 vaccination. Vaccine 2007; 25: 5958-67.

[70] $\mathrm{Hu} \mathrm{XD}, \mathrm{Yu} \mathrm{DH}$, Chen ST, et al. A combined DNA vaccine provides protective immunity against Mycobacterium bovis and Brucella abortus in cattle. DNA Cell Biol 2009; 28: 191-9.

(C) Oliveira et al.; Licensee Bentham Open.

This is an open access article licensed under the terms of the Creative Commons Attribution Non-Commercial License (http://creativecommons.org/licenses/by-nc/ $3.0 /$ ) which permits unrestricted, non-commercial use, distribution and reproduction in any medium, provided the work is properly cited. 